\title{
Thermal Stability and Decomposition of Perfluoroalkyl Substances on Spent Granular Activated Carbon
}

\author{
Feng Xiao*,§, Pavankumar Challa Sasis, Bin Yao ${ }^{\dagger}$, Alena Kubátová ${ }^{\dagger}$, Svetlana A. Golovko ${ }^{\ddagger}$, Mikhail \\ Y. Golovko ${ }^{\ddagger}$ Dana Soli§
}

§Department of Civil Engineering, University of North Dakota, 243 Centennial Drive Stop 8115, Grand Forks, North Dakota 58202, United States

†Department of Chemistry, University of North Dakota, 151 Cornell Street Stop 9024, Grand Forks, North Dakota 58202, United States

‡Department of Biomedical Sciences, University of North Dakota, 1301 Columbia Road North Stop 9037 , Grand Forks, North Dakota 58202, United States

\footnotetext{
*corresponding author contact information: Phone: +1-701-747-5150; fax: +1-701-777-3782; e-mail addresses: Feng.Xiao@engr.UND.edu; $\underline{\text { fxiaoee@gmail.com }}$
} 


\section{Table of Contents}

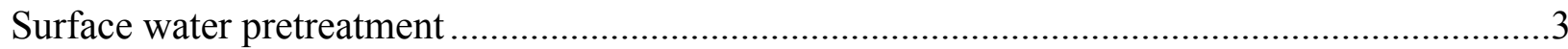

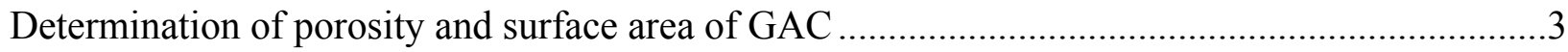

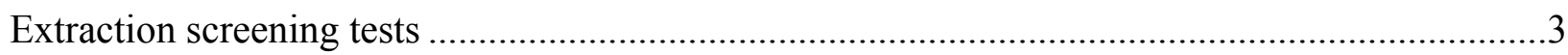

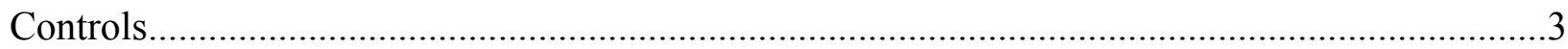

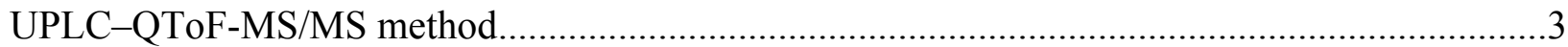

Thermal treatment in a closed system with air .............................................................

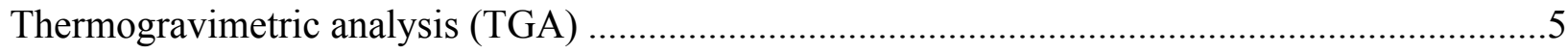

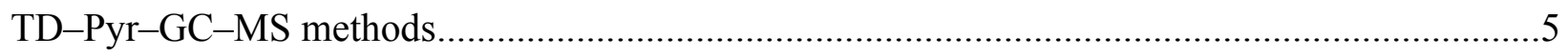

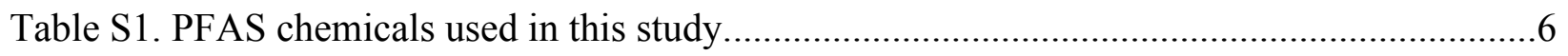

Figure S1. Pore size distribution of Filtrasorb 200 .......................................................

Figure S2. Thermal destabilization half-lives of PFOA and PFOS calculated from first-order rate constants.

Figure S3. TD-Pyr-GC-MS chromatograms (a-e) and mass spectra (f-i) of thermal decomposition products of PFOA at different temperatures and predetermined durations. 


\section{Surface water pretreatment}

Pretreatment included 1-min coagulation with alum $\left(\mathrm{Al}_{2}\left(\mathrm{SO}_{4}\right)_{3} \cdot 18 \mathrm{H}_{2} \mathrm{O}\right)$ at a dose of 40 $\mathrm{mg} / \mathrm{L}, 20$-min flocculation, 30-min settling, and filtration through a Whatman paper filter (Grade 5) to remove remaining fine particles/flocs. ${ }^{1}$

\section{Determination of porosity and surface area of GAC}

The porosity and pore size distribution (Figure S1) of GAC particles were measured by means of $\mathrm{N}_{2}$ porosimetry at $77 \mathrm{~K}$ (Autosorb-iQ, Quantachrome, Boynton Beach, FL) as detailed previously. ${ }^{2-4}$ The specific surface area of GAC was calculated by the 11-point Brunauer-Emmett-Teller (BET) method. The micro- and meso-porosities and pore size distributions of GAC were determined by quenched solid density functional theory from the $\mathrm{N}_{2}$ adsorption isotherm at $77 \mathrm{~K} .^{2-4}$

\section{Extraction screening tests}

In these test, GAC particles $(0.07 \mathrm{~g})$ in pre-cleaned polypropylene vials were spiked with a known volume of $1 \times 10^{-3} \mathrm{~mol} / \mathrm{L}$ PFAS stock solution in methanol. Then $10 \mathrm{~mL}$ of methanol, methanol with $100 \mathrm{mmol} / \mathrm{L} \mathrm{NaOH}$, or methanol with $100 \mathrm{mmol} / \mathrm{L} \mathrm{NH}_{4} \mathrm{Ac}$ was added to the tubes. The mixture was then ultrasonicated at room temperature or at $60^{\circ} \mathrm{C}$ for $30 \mathrm{~min}$ and subsequently centrifuged. Afterwards, the liquid phase was sampled and microfiltered $(0.45 \mu \mathrm{m}$ nylon filter; Thermo Scientific ${ }^{\mathrm{TM}}$ Target $\left.2^{\mathrm{TM}}\right)$. The concentration of PFAS in the filtrate was determined and the mass was calculated to determine the recovery by comparing it with the spiked mass.

\section{Controls}

The quartz tube was washed thoroughly after every thermal treatment of PFAS-containing GAC samples. In selected tests (at HTTs of 200, 300, 500, and $700{ }^{\circ} \mathrm{C}$ ), after the thermal treatment and cool down, we rinsed the tube with $100 \mathrm{~mL}$ F-free deionized water (DW) and measured PFAS in the rinsate; no measurable concentrations of target PFAS were found.

Furthermore, triplicate unspiked controls were prepared in sorbate-free vials with only GAC in the unspiked pretreated surface water to examine possible contamination of samples by PFAS and $\mathrm{F}^{-}$from laboratory apparatus. GAC particles collected from control vials were freezedried and heated at a HTT up to $900{ }^{\circ} \mathrm{C}$. F and remaining PFAS in off-gas and on GAC particles after the thermal treatment were measured in the procedures as described in the text. No measurable $\mathrm{F}^{-}$and PFAS were found in these control samples.

\section{UPLC-QToF-MS/MS method}

The analysis was carried out on a Waters Acquity ultrahigh pressure liquid chromatography (UPLC) system coupled with a Waters QToF-MS/MS ${ }^{1}$ (Synapt G2-S, Waters Corporation, Milford, MA, USA) available in the Department of Biomedical Sciences of University of North Dakota. Chromatography was performed using a Waters Acquity UPLC BEH Shield RP18 column $(100 \times 2.1 \mathrm{~mm} ; 130 \AA ; 1.7 \mu \mathrm{m})$ with a Waters Acquity UPLC BEH Shield RP18 VanGuard precolumn $(5 \times 2.1 \mathrm{~mm} ; 130 \AA ; 1.7 \mu \mathrm{m})$. The mobile phase consisted of eluent A ( $2 \mathrm{mM}$ ammonium 
formate in Optima ${ }^{\mathrm{TM}}$ water; LC/MS grade) and eluent $\mathrm{B}$ ( $2 \mathrm{mM}$ ammonium formate in Optima ${ }^{\mathrm{TM}}$ methanol; LC/MS grade). The elution started at 20\% B for 0.5 min and then was linearly increased to $85 \% \mathrm{~B}$ in $5 \mathrm{~min}$, further increased to $98 \% \mathrm{~B}$ in $0.1 \mathrm{~min}$ and kept isocratic for $1.5 \mathrm{~min}$. At 7.1 min, the $\mathrm{A} / \mathrm{B}$ ratio changed back to the initial value of $80 / 20$ over $0.1 \mathrm{~min}$ to re-equilibrate the column for another $1.3 \mathrm{~min}$. Analytes were eluted using a Waters Acquity UPLC pump equipped with a well-plate autosampler that was maintained at $8{ }^{\circ} \mathrm{C}$. The flow rate was maintained at 0.45 $\mathrm{mL} / \mathrm{min}$, and the column temperature was $55^{\circ} \mathrm{C}$. The UPLC retention times were $4.6,4.7$, and 4.8 min for PFOAAmS, PFOA, and PFOAB, respectively.

Mass spectrometry analysis was performed using the Synapt G2-S QToF-MS with an ESI source operated in a negative ion mode. MS operating conditions were as follows: cone voltage, $20 \mathrm{~V}$; capillary voltage, $1.8 \mathrm{kV}$; source temperature, $110{ }^{\circ} \mathrm{C}$; desolvation temperature, $350{ }^{\circ} \mathrm{C}$; cone gas flow rate, $10 \mathrm{~L} / \mathrm{h}$; and desolvation gas flow, $1,000 \mathrm{~L} / \mathrm{h}$. The analyzer was operated with an extended dynamic range at 10,000 resolution (fwhm at $\mathrm{m} / \mathrm{z} 554$ ) with an acquisition time of $0.1 \mathrm{~s}$. The Synapt G2-S ToF MS ${ }^{\mathrm{E}}$ mode was used to collect data with the T-wave element alternated between a low energy of $2 \mathrm{~V}(\mathrm{MS})$ and high energy $\left(\mathrm{MS}^{\mathrm{E}}\right)$ states in which the transfer T-wave element voltage ranged from 10-25 V. Leucine enkephalin $(400 \mathrm{pg} / \mu \mathrm{L})$ was infused at a rate of $10 \mu \mathrm{L} / \mathrm{min}$ for mass correction. MassLynx V4.1 software (Waters) was used for instrument control, acquisition, and mass analysis. The structural information of the degradation products was obtained by the state-of-the-art $\mathrm{MS}^{\mathrm{E}}$ function that allows the simultaneous acquisition of both MS and MS/MS fragmentation during a single chromatographic run.,

Quantification of all target PFAAs were made using extracted ion currents (10 ppm mass window) and were based on their $\mathrm{m} / \mathrm{z}$ values and UPLC retention times relative to a six-point external calibration standard curves.

\section{Thermal treatment in a closed system with air}

Certain reactivation experiments were also performed in an air atmosphere in a muffle furnace (Neytech., Vulcan 3-550, USA) at a predetermined HTT. For these experiments, PFASloaded GAC particles were placed in borosilicate biochemical oxygen demand (BOD) bottles with glass pennyhead stoppers, and heated in an air atmosphere in the muffle furnace at a predetermined HTT. After heat treatment and cool down, $50 \mathrm{~mL}$ F-free DW or $10 \mathrm{~mL}$ methanol (amended with $100 \mathrm{mmol} / \mathrm{L}$ ammonium acetate) was added to the bottle and ultrasonicated for $30 \mathrm{~min}$. Then, we measured concentrations of $\mathrm{F}^{-}$in the aqueous solution as well as PFAS in methanol.

To test the extent to which BOD bottles were leakproof, we spiked known volumes of sodium fluoride stock solution $(10 \mathrm{mg} / \mathrm{L})$ to three groups of raw GAC particles, freeze-dried the GAC particles, placed them in triplicate BOD bottles, and heated them in the muffle furnace at a HTT of 400,450 and $500{ }^{\circ} \mathrm{C}$. After heat treatment and cool down, $50 \mathrm{~mL}$ F-free DW was added to the bottle and ultrasonicated for $30 \mathrm{~min}$. Then, we determined concentration and mass of $\mathrm{F}^{-}$in solution and compared with the spiked mass. The difference varied by $<14 \%$, generally considered to be within the range of analytical variability. In some cases, cracks on the stopper occurred after moving the bottle from 450 or $500{ }^{\circ} \mathrm{C}$ in the muffle furnace (suddenly) to room temperature. Even with these cracks, we did not observe apparent loss of $\mathrm{F}$ from the bottle. 


\section{Thermogravimetric analysis (TGA)}

We also conducted TGA of PFAS chemical powders using a thermal analyzer system (TGA/STD Q600, TA Instrument, DE) in an atmosphere of $\mathrm{N}_{2}, \mathrm{CO}_{2}$, or $\mathrm{O}_{2}$. In one instance, we performed dynamic TGA scans from room temperature to a predetermined temperature. The other TGA experiment was devoted to calculation of the weight loss of PFAS under isothermal conditions. In brief, we conducted a scan from room temperature to the predetermined temperature in which the sample was kept under isothermal conditions.

\section{TD-Pyr-GC-MS methods}

PFOA thermal decomposition products were analyzed by a thermal desorption-pyrolysis system (CDS Analytical) connected to a gas chromatograph-mass spectrometer system (Agilent GC 7890 and 5975C MS; Santa Clara, CA) (TD-Pyr-GC-MS). The TD-Py program consisted of single or sequential thermal steps with the pyroprobe heated for $30 \mathrm{sec}$ at thermal desorption temperatures of $200,300{ }^{\circ} \mathrm{C}$, and pyrolysis temperatures of 400,500 , and $10 \mathrm{sec}$ at $890{ }^{\circ} \mathrm{C}$ with the interface heated to $300{ }^{\circ} \mathrm{C}$ and $350{ }^{\circ} \mathrm{C}$, respectively. For each temperature the interface was opened for $2.5 \mathrm{~min}$ to transfer the analytes (products) through the heated transferline, a GC injector port (in $10: 1 \mathrm{split}$ ) kept at $300{ }^{\circ} \mathrm{C}$ onto the column held at $40{ }^{\circ} \mathrm{C}$, this was then followed by GC analysis. The $\mathrm{GC}$ temperature program started at $40^{\circ} \mathrm{C}$ for 1 min followed by a gradient of $40^{\circ} \mathrm{C} / \mathrm{min}$ to $80^{\circ} \mathrm{C}$, and then $25^{\circ} \mathrm{C} / \mathrm{min}$ to $320^{\circ} \mathrm{C}$, and held for $4 \mathrm{~min}$. The $\mathrm{GC}$ was equipped with a $52.5 \mathrm{~m}$ HP-5MS column $(0.25 \mu \mathrm{m}$ film thickness and $0.25 \mathrm{~mm}$ i.d.). The MS analysis was performed with electron ionization in mass range $35-850 \mathrm{~m} / \mathrm{z}$. Ultra-pure helium $(99.999 \%)$ was used as the carrier gas with a constant flow rate of $1.1 \mathrm{~mL} \mathrm{~min}^{-1}$. 


\section{Tables}

Table S1. PFAS chemicals used in this study.

\begin{tabular}{|c|c|c|c|}
\hline PFCAs & Acronyms & Purity & Providers \\
\hline Perfluorobutyric acid (C4) & PFBA & $\geq 99.5$ & Sigma-Aldrich \\
\hline perfluoropentanoic acid (C5) & PFPeA & $97 \%$ & Sigma-Aldrich \\
\hline Perfluoroheptanoic acid (C6) & PFHpA & $\geq 98 \%$ & Fisher Scientific \\
\hline Perfluorooctanoic acid (C8) & PFOA & $95 \%$ & Sigma-Aldrich \\
\hline Perfluorononanoic acid (C9) & PFNA & $97 \%$ & Sigma-Aldrich \\
\hline Perfluorodecanoic acid (C10) & PFDA & $98 \%$ & Sigma-Aldrich \\
\hline perfluoroundecanoic acid (C11) & PFUnDA & $95 \%$ & Sigma-Aldrich \\
\hline PFSAs & Acronyms & Purity & Provider \\
\hline $\begin{array}{l}\text { Perfluorobutanesulfonic acid } \\
\text { potassium salt }(\mathrm{C} 4)\end{array}$ & PFBS & $98.0 \%$ & Sigma-Aldrich \\
\hline $\begin{array}{l}\text { Perfluorohexanesulfonic acid } \\
\text { potassium salt (C6) }\end{array}$ & PFHxS & $\geq 98.0 \%$ & Sigma-Aldrich \\
\hline $\begin{array}{l}\text { Perfluorooctanesulfonic acid } \\
\text { potassium salt }(\mathrm{C} 8)\end{array}$ & PFOS & $\geq 98.0 \%$ & Sigma-Aldrich \\
\hline $\begin{array}{c}\text { PFECA } \\
\text { (perfluoroalkyl ether } \\
\text { carboxylic acid) }\end{array}$ & Acronym & Purity & Provider \\
\hline $\begin{array}{l}\text { 2,3,3,3-tetrafluoro-2- } \\
(1,1,2,2,3,3,3- \\
\text { heptafluoropropoxy)propanoic } \\
\text { acid (C3) }\end{array}$ & HFPO-DA (GenX) & $97 \%$ & Fisher Scientific \\
\hline
\end{tabular}




\section{Figures}

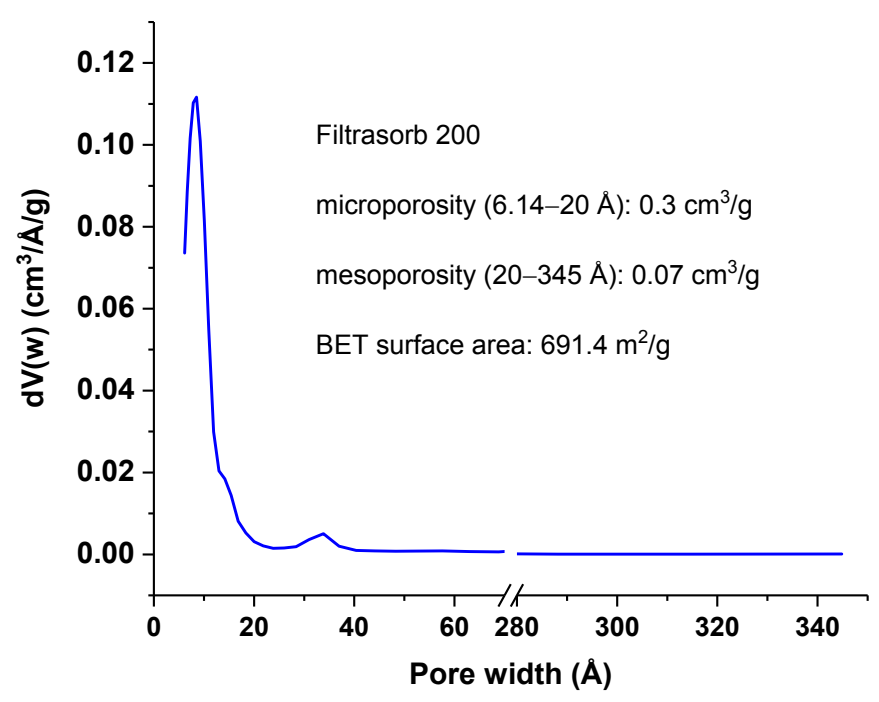

Figure S1. Pore size distribution of Filtrasorb 200.

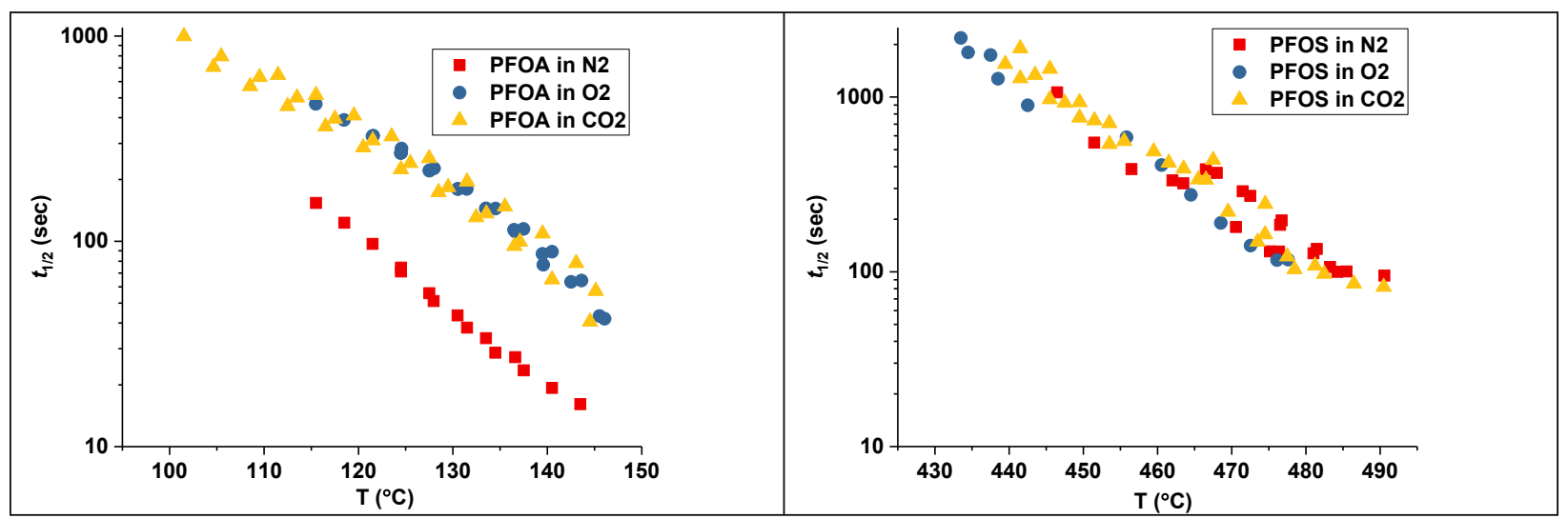

Figure S2. Thermal destabilization half-lives of PFOA and PFOS calculated from first-order rate constants. 


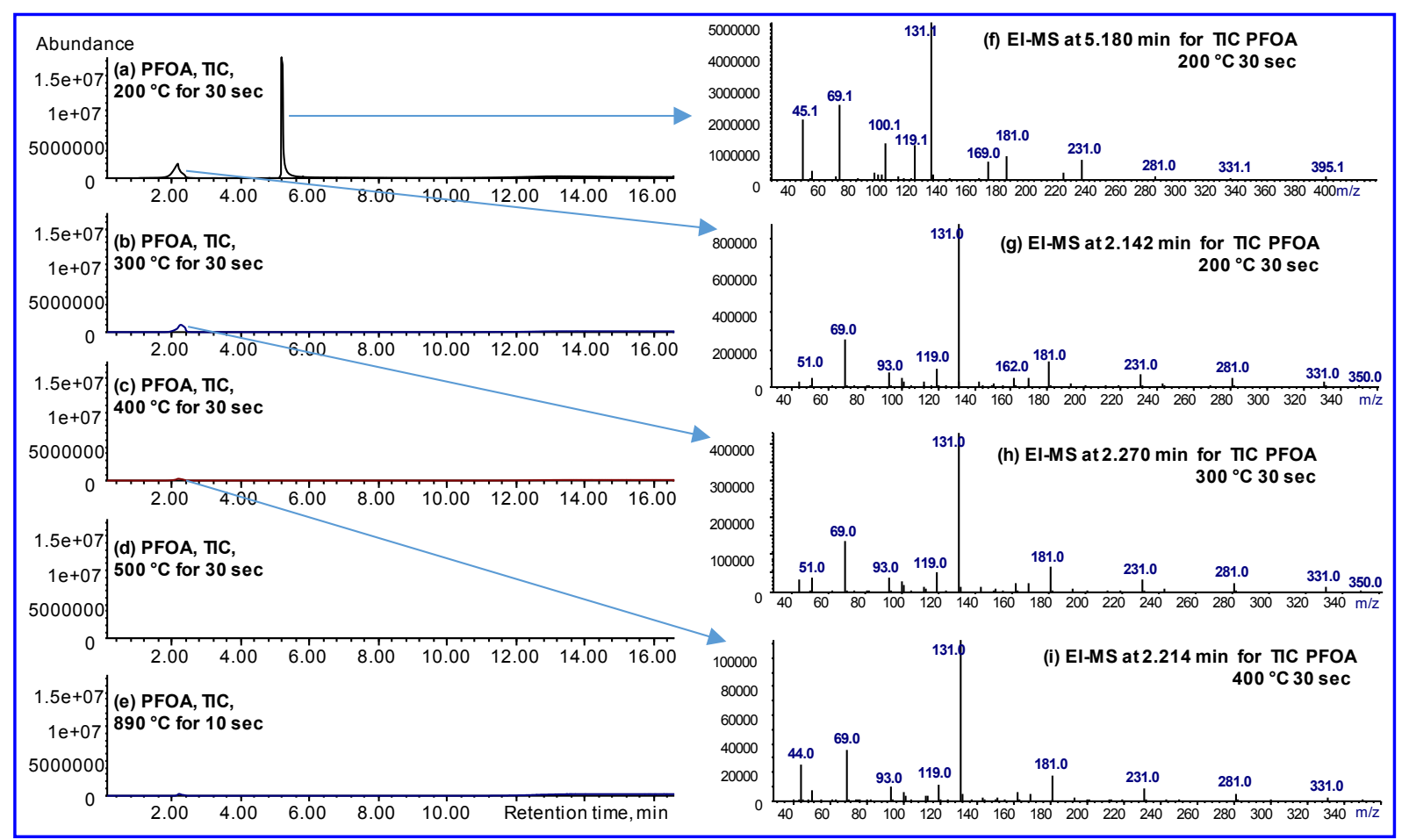

Figure S3. TD-Pyr-GC-MS chromatograms (a-e) and mass spectra (f-i) of thermal decomposition products of PFOA at different temperatures and predetermined durations.

\section{References for SI}

(1) Xiao, F.; Hanson, R. A.; Golovko, S. A.; Golovko, M. Y.; Arnold, W. A., PFOA and PFOS are generated from zwitterionic and cationic precursor compounds during water disinfection with chlorine or ozone. Environ Sci Tech Let 2018, 5, 382-388.

(2) Xiao, F.; Gámiz, B.; Pignatello, J. J., Adsorption and desorption of nitrous oxide by raw and thermally air-oxidized chars. Sci. Total Environ. 2018, 643, 1436-1445.

(3) Xiao, F.; Bedane, A. H.; Zhao, J. X.; Mann, M. D.; Pignatello, J. J., Thermal air oxidation changes surface and adsorptive properties of black carbon (char/biochar). Sci Total Environ 2017, 618, 276-283.

(4) Cao, X. Y.; Xiao, F.; Duan, P.; Pignatello, J. J.; Mao, J. D.; Schmidt-Rohr, K., Effects of post-pyrolysis air oxidation on the chemical composition of biomass chars investigated by solid-state nuclear magnetic resonance spectroscopy. Carbon 2019, 153, 173-178.

(5) Waters., An overview of the principles of $\mathrm{MS}^{\mathrm{E}}$, the engine that drives $\mathrm{MS}$ performance. In Waters Corporation: Milford, MA, 2011; pp 1-6.

(6) Xiao, F.; Golovko, S. A.; Golovko, M. Y., Identification of novel non-ionic, cationic, zwitterionic, and anionic polyfluoroalkyl substances using UPLC-TOF-MS(E) highresolution parent ion search. Anal Chim Acta 2017, 988, 41-49. 